\title{
Rapid Atomic-Resolution Image Analysis: Towards Near-Instant Feedback
}

Oleg S. Ovchinnikov, ${ }^{1,2}$ Andrew O'Hara, ${ }^{1}$ Sergei V. Kalinin, ${ }^{3,2}$ Stephen Jesse, ${ }^{3,2}$ Albina Y. Borisevich, ${ }^{4,2}$ Sokrates T. Pantelides ${ }^{1}$

1. Department of Physics and Astronomy, Vanderbilt University, Nashville, TN, USA

2. Institute for Functional Imaging of Materials, Oak Ridge National Laboratory, Oak Ridge, TN, USA

3. Center for Nanophase Materials Sciences, Oak Ridge National Laboratory, Oak Ridge, TN, USA

4. Materials Science and Technology Division, Oak Ridge National Laboratory, Oak Ridge, TN, USA

Over the last two decades the capabilities of electron microscopes in delivering data on materials behavior at high spatial and energy resolution have grown at breakneck speed. It has recently become clear that, along with the ability to acquire such datasets, the ability to analyze the data to extract all the relevant physical information is just as critical to realizing the full potential of the advanced imaging techniques. A wide selection of post-processing approaches can be deployed to uncover the distribution of quantitative materials parameters across the field of view of the image. However, most algorithms require user input on multiple steps and run with speeds substantially slower than acquisition. At the same time, there is an obvious value in providing near-instant, input-less feedback to the microscope operator so that the subsequent experiments can be better targeted to regions and features of interest.

In this paper we will present results on the development of rapid unsupervised algorithms for analysis of atomic-resolution STEM images. We are able to demonstrate that, using only the information in the image tags, a rapid algorithm based on a whole-image transform can identify atomic columns in 1 sec or less on an average desktop-style computer. Subsequently, a rapid, graph-theory-based approach can reliably identify (also in $1 \mathrm{sec}$ or less) a variety of structural defects in the identified atomic grouping, with no prior knowledge of the lattice type(s) present and no requirement to continuity thereof. Preliminary results indicate that substitutional defects, which often result in local lattice disturbance, can also be successfully identified with this approach. Fig 1 shows an example of a STEM image (a) and the corresponding output with atomic columns marked and distinct atomic environments color-coded (b).

Beyond rapid feedback during acquisition, the unsupervised analysis approach developed in this work could be used to identify interesting images for further analysis, compute dataset-wide statistics on prevalence of atomic configurations, and potentially test hypotheses generated during in-depth analysis of individual images over a wider dataset. Remarkably, this analysis can also be deployed to provide input for theoretical calculations such as Density Functional Theory studies, which can, for example, determine not only the distribution of atomic columns within the image plane, but also find full 3D coordinates of atoms via total-energy minimization. We carried out a proof-of-principle study for image analysis/DFT hybrid approach using STEM images of misoriented bilayer graphene to fully characterize its 3D structure and quantify nanoscale ripples present in the system. Due to the unsupervised character of our approach, it could potentially become a basis for an iterative image-analysis-DFT-simulation loop, enabling us to perform data to simulation comparison on a qualitatively new level. Ideas for acquisition workstation architecture that would enable instantaneous feedback setup will also be discussed [1].

\section{References:}

[1] Work performed at Vanderbilt was supported in part by Department of Energy grant DE-FG0209ER46554 (OOS, AOH, STP). This work was performed in part at the Center for Nanophase Materials 
Sciences, which is supported at Oak Ridge National Laboratory by Scientific User Facilities Division, US DOE. OOS, SJ and SVK were also supported by ORNL's Labotatory Directed Research and Development Fund. AYB was supported by the Division of the Materials Science and Engineering, US DOE.

(a)

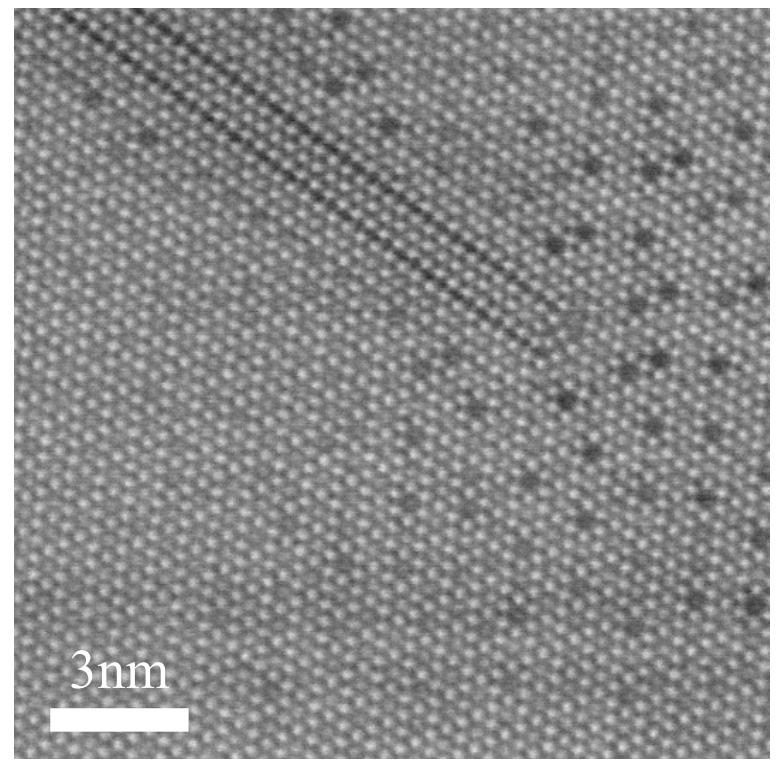

(b)

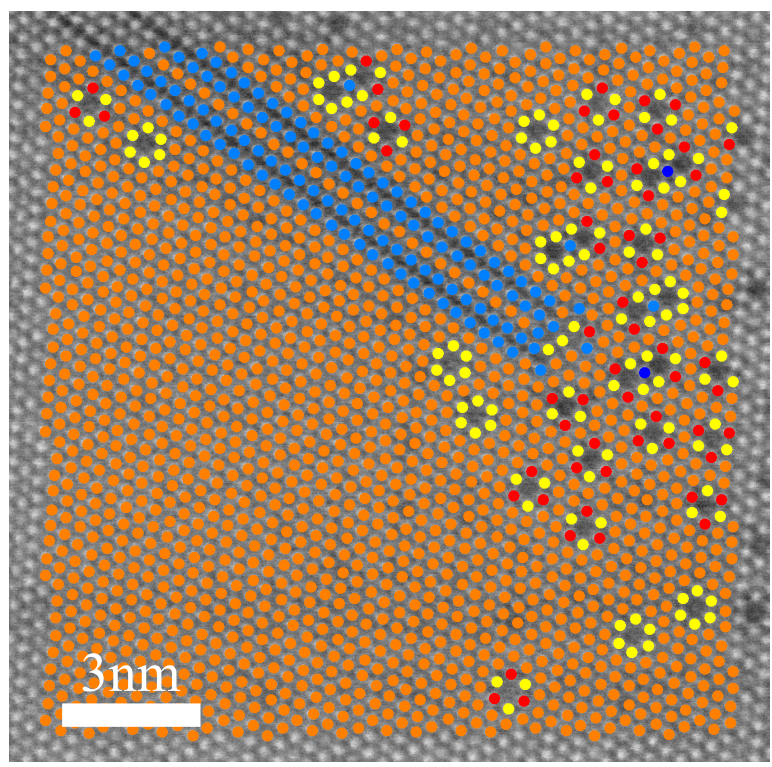

Figure 1. An example of the information returned by rapid unsupervised image analysis within $\sim 2$ seconds: (a) input HAADF STEM image (b) returned image with atomic columns marked and distinct atomic environments color coded. 